\title{
Norois
}

Environnement, aménagement, société

$217 \mid 2010 / 4$

Interroger les processus de valorisation des espaces urbains

\section{Une patrimonialisation sans appropriation ? Le cas de l'architecture de la reconstruction au Havre}

A patrimonialisation without appropriation? The case of the architecture of reconstruction of Le Havre

\section{Maria Gravari-Barbas et Cécile Renard}

\section{(2) OpenEdition}

\section{Journals}

\section{Édition électronique}

URL : https://journals.openedition.org/norois/3476

DOI : $10.4000 /$ norois.3476

ISBN : 978-2-7535-1565-9

ISSN : 1760-8546

Éditeur

Presses universitaires de Rennes

Édition imprimée

Date de publication : 15 décembre 2010

Pagination : $57-73$

ISBN : 978-2-7535-1340-2

ISSN : 0029-182X

Référence électronique

Maria Gravari-Barbas et Cécile Renard, « Une patrimonialisation sans appropriation ? Le cas de l'architecture de la reconstruction au Havre », Norois [En ligne], 217| 2010/4, mis en ligne le 31 décembre 2012, consulté le 07 décembre 2022. URL : http://journals.openedition.org/norois/3476 ; DOI : https://doi.org/10.4000/norois.3476 


\title{
UNE PATRIMONIALISATION SANS APPROPRIATION? \\ Le cas de l'architecture de la reconstruction au Havre
}

\author{
Maria Gravari-Barbas, Cécile Renard \\ EA EIREST \\ (Université de Paris 1 Panthéon Sorbonne), \\ 12 place du Panthéon - 75005 Paris, France \\ maria.gravari-barbas@wanadoo.fr, cecile.renard@malix.univ-paris1.fr
}

\section{RÉSUMÉ}

L'architecture de reconstruction du Havre, ouvre de l'architecte Anguste Perret, inscrite sur la liste du patrimoine mondial de l'Unesco en 2005, est un exemple intéressant d'une patrimonialisation orchestrée par les experts (nationaux et internationaux) et le pouvoir local: les premiers étant conscients de la valeur intrinsèque de l'œuvre de Perret et de la place qu'elle occupe dans l'histoire de l'architecture contemporaine; les seconds étant persuadés que l'inscription du Havre pourrait permettre de disposer d'un outil de valorisation et de reconstruction identitaire de cette ville-martyre.

Dans ce processus de patrimonialisation du Havre, que nous pouvons qualifier de topdown, la population Havraise a été quasiment absente. Ce processus correspond par conséquent à une dynamique portée par des acteurs extérieurs et instrumentalisée par le politique, mais dans laquelle les résidents ont joué un rôle marginal.

Nous avons souhaité nous poser la question de l'évolution de ce schéma de patrimonialisation. La littérature de plus en plus abondante sur le patrimoine mondial semble confirmer que celui-ci agit comme un formidable moteur à différents niveaux : économique, identitaire, touristique et ce sont ces entrées que nous avons explorées dans le cadre de cet article. Nous faisons l'hypothèse d'une patrimonialisation sans appropriation qui, cinq ans après l'inscription, continue à fonctionner par l'intermédiaire du regard extérieur : le regard touristique tend aujourd'hui à succéder au regard des experts.

MotS CLÉ : Patrimonialisation - appropriation - Le Havre - tourisme urbain - architecture - reconstruction

\footnotetext{
ABSTRACT

A patrimonialisation without appropriation? The case of the architecture of reconstruction of Le Havre

The architecture of reconstruction of Le Havre, work of the architect Anguste Perret, inscribed in 2005 at the Unesco World Heritage list, is an interesting example of heritage building orchestrated by national and international experts and by the local power: the first being aware of the merits of Perret's architecture and the latter convinced that Le Havre listing could provide a recovery tool for the reconstruction of the identity of this martyr city.

In this process, we can qualify for top-down, Le Havre residents was virtually absent. Le Havre heritage-building process is therefore an impulse coming from "outsiders" and instrumented by the local power, but in which residents have played a marginal role.
} 
We wished in this paper to examine the evolution of this heritage-building schema. A more and more abundant literature on World Heritage seems to confirm that the latter acts as a-powerful and positive engine at different levels: economic, identity, tourism; these are the entries we explore in this paper. We make the hypothesis of a heritage-building process without appropriation that, 5 years after the listing, continues to operate thanks to the external insight: the tourist gaze today tends to succeed to the one of the experts.

KEY WORDS : Heritage-building - appropriation - Le Havre - urban tourism - architecture - post-2 ${ }^{\text {nd }}$-World-Word-reconstruction

En juin 2010, la ville du Havre a fêté le $5^{\mathrm{e}}$ anniversaire de son inscription sur la liste prestigieuse du patrimoine mondial de l'Unesco. Que de chemin parcouru dans un laps de temps aussi court! À peine cinq ans auparavant, les journaux s'étonnaient de la décision de l'Unesco de classer « cette sinistre caricature de la reconstruction d'après guerre, ce béton âpre, cette ville au carré ${ }^{1}$ ». " L'Unesco est-elle tombée sur la tête? » (Martin-Chauffier, 2005) s'interrogeait Paris-Match magazine. Autant dire que l'annonce de l'inscription du centre reconstruit du Havre sur la très convoitée liste du patrimoine mondial de l'Unesco, avait été accueillie avec scepticisme. Le Havre correspond en effet à l'exemple idéal-typique d'une ville reconstruite après la seconde guerre mondiale, très éloigné de la représentation consensuelle, toujours aujourd'hui, de la ville historique. Surnommée «Stalingrad-sur-Mer », traversée par la «Staline-allée ${ }^{2}$ », choisie comme lieu de tournage de films restituant l'ambiance des villes de l'Est à l'époque du rideau de fer ${ }^{3}$, le patrimoine urbain du Havre est radicalement différent de celui des autres villes françaises du patrimoine mondial, telles Lyon, Bordeaux ou Arles.

Par quels cheminements cette ville mal- aimée a-t-elle réussi cet exploit spectaculaire? Quels ont été les acteurs de cette construction patrimoniale? Et de quelle manière, une fois cette reconnaissance acquise, a-t-elle été appropriée par les populations locales qui, contrairement aux schémas classiques, ont joué un rôle plutôt marginal dans la patrimonialisation du Havre? Y a-t-il eu une appropriation de cette patrimonialisation spectaculaire du Havre? L'approche top-down qui a prévalu dans l'instrumentalisation du patrimoine Perret a-t-elle été suivie par des approches patrimonialisantes de type bottom-up?

Cet article vise à analyser, cinq ans après l'inscription du Havre sur la liste du patrimoine mondial, les voies et les cheminements d'une patrimonialisation sans appropriation locale, ce qui n'est pas sans poser des questions quant à la nature du patrimoine et du rôle que celui-ci est appelé à jouer dans l'évolution de la ville.

Nous nous appuierons pour cela sur une analyse critique : d'une part du dispositif qui a été mis en place par la ville et les services en charge du patrimoine; d'autre part sur la manière dont ces mesures et projets sont reçus par les populations locales et les touristes. Une enquête qualitative menée entre février et avril 2010 dans l'appartement témoin d'Auguste Perret a permis de recueillir des avis et des positionnements de visiteurs. Elle a été complétée par des entretiens avec plusieurs acteurs locaux (Ville d'Art et d'Histoire [Ville d'A + H], FISAC ${ }^{4}$, ZPPAUP $^{5}$ ) et avec les associations des commerçants qui ont permis de confronter les projets

1. Télérama, n² 2909, 12 octobre 2005, p. 69.

2. Le boulevard Foch, un des axes principaux de l'ordonnancement de Perret, menant de l'Hôtel de ville vers la Porte Océane, a reçu le surnom évocateur de «Staline-allée », tandis que Le Havre - «Stalingrad-sur-mer » - était assimilée à une ville du bloc de l'Est. Ces commentaires étaient inspirés à la fois de l'austère architecture en béton et de l'équipe communiste qui a gouvernée la ville pendant presque 40 ans (1956-1995).

3. En 1988 le centre-ville du Havre sert de décor au film d’Agnieska Holland, «Le complot », inspiré de l'un des épisodes les plus dramatiques de la répression contre Solidarnosc, l'assassinat du père Jerzy Popieluszko.

4. Fonds d'intervention pour les services, l'artisanat et le commerce.

5. Zone de protection du patrimoine architectural, urbanistique et paysager. 
lancés avec leur réception par les publics, locaux et touristiques. Finalement, l'analyse des enquêtes sociologiques produites par l'équipe du CERLIS (Monjaret et al., 2010), recueillant les avis des anciens habitants du Havre, ont apporté des éclairages précieux.

Cet article s'inscrit dans le prolongement de plusieurs autres travaux menés sur la question par M. Gravari-Barbas (2002, 2003, 2004a,b, 2006, 2008a,b) et par plusieurs chercheurs (Barot, 1994; Maubant, 1994 ; Jacono et Arnould 2000) qui se sont intéressés à la patrimonialisation du Havre. Il vise à actualiser l'état des connaissances sur la question, cinq ans après l’inscription.

\section{Une patrimonialisation instrumentalisée}

Loin d'être le résultat d'une reconnaissance «spontanée » de la part des experts de l'Unesco, l'inscription du Havre sur la liste du patrimoine mondial représente au contraire le fruit de démarches menées par les acteurs Havrais, assistés par des experts et fortement appuyées diplomatiquement par l'État Français.

La reconnaissance de la ville par l'Unesco revêt un caractère emblématique, puisqu'il s'agit de la première ville reconstruite selon un plan moderne à figurer sur la prestigieuse liste ${ }^{6}$. Il est connu que la compétition pour y figurer est devenue acharnée au cours des dernières années. Les retombées touristiques, médiatiques et économiques, sont supposées être aussi importantes que celles d'autres grands événements, manifestations ou labellisations internationales - même si les études produites sur la question proposent des lectures plus nuancées (Gravari-Barbas et Jacquot, 2008). Le Comité du patrimoine mondial tend à être aussi sélectif et exclusif que le Comité Olympique; il reçoit des propositions de plus en plus nombreuses et de mieux en mieux formulées et argumentées.

Cet effort de lobbying actif est révélateur de l'importance que le label revêt désormais aux yeux des acteurs locaux et du public. Il marque en effet souvent l'entrée de la ville dans une démarche de mise en valeur construite et structurée de ses ressources patrimoniales. Le développement économique des villes repose en effet désormais de plus en plus sur leur capacité de se distinguer, de se singulariser. Le label Unesco, «produit-monde », par définition et par excellence $^{7}$, représente une marque à haute valeur rajoutée, souvent instrumentalisée en ce sens. Dans le cas du Havre, la reconnaissance internationale est adressée aussi, par un effet de miroir, à la population locale en tant que moyen de (re)construction d'une identité forte.

\section{LA RECONNAISSANCE DE L'UNESCO, « PLANCHE DE SALUT » D’UNE VILle SINISTRÉE?}

Contrairement à d'autres villes reconstruites en Europe (Gravari-Barbas, 2004b), le ministère français de la Reconstruction a opté pour un plan radicalement moderne, application à grande échelle des principes novateurs sur la quasi-totalité de la zone touchée par les bombardements (Abram, 1997-1998).

En effaçant le passé, le nouveau plan d'Auguste Perret a doté la ville, qui figurait jusqu'alors parmi les villes françaises les plus insalubres, d'un urbanisme digne des CIAM ${ }^{8}$. L'architecte «a transformé Le Havre en une ville-monument aux espaces auliques, capables de se mesurer à la grandiose métropole parisienne dont elle est devenue la porte sur l'océan... » (Gargiani, 1996).

6. Si elle n'est ni la première ville «moderne » à y figurer (la ville de Brasilia a été classée dès 1987) ni la première ville « reconstruite » (Varsovie a été classée en 1980), elle est la première qui allie ces deux caractéristiques, modernité et reconstruction.

7. Dans le sens d'une production symbolique tirant sa substance du contexte mondial et à consommation mondiale, créé en référence à un ensemble de biens existant de par le monde.

8. Les congrès internationaux d'architecture moderne ou CIAM, sont des réunions internationales, organisées entre 1928 et 1956, nées du besoin de promouvoir une architecture et un urbanisme fonctionnels. Le CIAM de 1933 a produit un document doctrinal fondateur de l'architecture moderne fonctionnelle, la Charte d'Athènes. 
Dans les années qui ont suivi la reconstruction, et au moins jusqu'aux années 1970, Le Havre a nationalement projeté l'image de la modernité. Modernité architecturale certes, mais aussi modernité du mode de vie dans ces nouveaux appartements qui invitaient à vivre autrement, et qui ont été à l'origine de la réinvention du quotidien ou de l'évolution de l'espace domestique et du mobilier (Colliard, 1998). La crise économique qui frappe de plein fouet Le Havre dès le début des années 1970 signifie la fin de l'aventure de la modernité. La fermeture des grands sites métallurgiques comme Alstom et des évolutions liées aux usines Renault ont durement touché l'économie locale. Mais le choc principal est la fermeture en 1999 des Ateliers et Chantiers du Havre, industrie qui concernait plus de 2500 employés. La fermeture a marqué la fin - après pratiquement 400 ans d'histoire - de l'activité la plus ancienne et la plus profondément établie au Havre. Le chômage, la récession, la crise sociale ont profondément marqué l'identité de la ville qui, pendant des décennies, a vécu en étroite symbiose avec son port (Banzet, 2001). Cette crise sociale et économique a "pénétré » la ville, son ambiance et son environnement bâti, en créant un amalgame entre la ville, son cadre, son économie. Elle a contribué à une forte dépréciation de l'architecture de la reconstruction, devenue le réceptacle et l'élément le plus visible de la déchéance de la ville.

\section{LA LABELLISATION UNESCO, FRUIT D'UN LOBBYING INTENSE}

La labellisation de l'Unesco intervient dans un contexte économique et social local a priori peu propice, dans lequel la construction d'un projet pérenne de patrimonialisation a imposé au préalable la déconstruction des partis-pris, des représentations, des pratiques qui marquaient la ville.

Comprendre l'inscription du Havre sur la liste de l'Unesco implique ainsi de se situer à la croisée de tendances nationales ou internationales et locales, de suivre par conséquent la manière dont le système d'acteurs qui a œuvré dans le classement de la ville a pris en compte les évolutions sociétales plus générales (et notamment l'élargissement de la notion du patrimoine vers l'architecture moderne) ainsi que la manière dont il les a, localement, traduites en actes.

L'évolution en termes de reconnaissance de l'architecture du $\mathrm{Xx}^{\mathrm{e}}$ siècle et plus particulièrement celle de la reconstruction, timidement esquissée sur le plan national à la fin du $\mathrm{Xx}^{\mathrm{e}}$ siècle, a été relayée de manière différenciée sur le plan local. Dans le cas du Havre qui représente aujourd'hui l'exemple le plus abouti de la reconnaissance de l'héritage de la reconstruction, la mise en évidence progressive sur le plan local du « patrimoine Perret » peut être définie comme une « réaction d'opportunité » de la part des acteurs locaux à un ensemble de contextes nationaux et internationaux qui lui étaient favorables. Davantage qu'une mise en place d'une stratégie conçue dès le départ comme un "scénario » de reconnaissance patrimoniale, on assiste à un ensemble d'actions, souvent prises par des acteurs différents voire opposés (y compris par deux équipes municipales successives ayant des valeurs et représentations patrimoniales différentes) mais qui, dans le moyen terme (et au moins depuis le début des années 1990 et jusqu'à la fin des années 2010) s'enchaînent dans la continuité.

Dans cet enchaînement des choses, on peut toutefois identifier un « avant » (équipe communiste) et un « après » 1995 (date qui correspond à la prise de commandes de la ville par l'équipe de droite dirigée par le maire Antoine Rufenacht). Si l'ancienne opposition de droite a longtemps décrié cette architecture, après les élections, le maire « a immédiatement saisi le bénéfice symbolique (réconcilier les Havrais avec leur ville) et le gain politique » au point de considérer le classement Unesco comme « le couronnement de [s]a carrière ${ }^{9}$ ».

Une fois les premières réactions consommées, la nouvelle équipe municipale a en effet assumé la réalité architecturale du Havre en l'interprétant non plus comme un handicap, 
mais comme un avantage - voire même le seul créneau porteur du Havre. Autrement dit, à partir du milieu des années 1990, la mise en valeur du " gisement » patrimonial de Perret devient, dans le contexte des évolutions sociétales présentées préalablement et grâce à un ensemble de personnalités « extérieures » œuvrant au sein du Ministère de la Culture, des instances architecturales internationales ou des universités, l'option officiellement adoptée par les acteurs locaux. Si donc ceux-ci n'ont pas été à l'origine de la découverte de ce « gisement ", ils ont acté les messages venus de l'extérieur argumentant pour l'intérêt intrinsèque de l'architecture Perret.

Les différentes mesures progressivement adoptées par les acteurs locaux (ZPPAUP : zone de protection du patrimoine architectural, urbain et paysager en $1995^{10}$, Ville d'Art et d'Histoire en 2001) témoignent des nouvelles ambitions de l'équipe municipale pour le patrimoine Perret (Gravari-Barbas, 2002, 2003).

La réussite de l'inscription sur la liste du patrimoine mondial couronne ainsi un long processus commencé discrètement dès 1994, sous l'ancienne équipe municipale, essentiellement sous l'impulsion de certains membres du DoCoMoMo ${ }^{11}$. En 2001 Le Havre franchit la première étape : elle est inscrite sur la liste des biens français soumise à l'Unesco. La candidature du Havre devient alors une « raison d'État ». Le dossier reçoit l'appui du Président de la République et par conséquent des deux ministres de la Culture successifs, ainsi que de l'Ambassadeur de France à l'Unesco. Le suivi politique du dossier a été continu : « des mois et des saisons au cours desquels les délégués de l'Unesco (étaient) homéopathiquement mais systématiquement tenus au courant de l'intérêt de la candidature du Havre ${ }^{12}$ ».

Aucune autre candidature française n'avait été présentée entre 2002 et 2004; en 2005 la candidature du Havre supplante celle de Bordeaux dont les quais prestigieux du XVIII ${ }^{\mathrm{e}}$ siècle étaient proposés au classement par le maire et ancien Premier ministre, Alain Juppé. Le choix de l'architecture de Perret, a priori peu évident ${ }^{13}$ se situe au croisement de considérations locales et nationales et témoigne d'un ensemble d'arbitrages pris à des niveaux différents. Elle signifie la consécration du patrimoine Perret. Celui-ci devient conjointement la carte de visite la plus parlante de la ville, son projet le plus identifiable et le tremplin pour la réalisation de ses futurs projets urbains.

\section{Un avenir urbain construit sur le patrimoine Perret}

La ville cherche à capitaliser sur la reconstruction et l'Unesco de manière plus générale, afin de construire un projet pérenne, non seulement architectural et urbanistique mais aussi humain, social et économique. L'inscription sur la liste du Patrimoine mondial de l'Unesco a incontestablement eu un effet bénéfique sur la confiance locale eu égard aux projets futurs de la ville. Si l'inscription a pu se réaliser, pourquoi d'autres projets ne le pourraient pas également?

Il semble justifié de mettre en rapport la reconnaissance du patrimoine du centre reconstruit avec la renaissance des docks du XIX ${ }^{\mathrm{e}}$ siècle et des quartiers est de la ville. Le futur grand projet du Havre, baptisé Centre de la Mer et du Développement Durable/Odyssey 21, pensé comme le «pendant culturel et urbain » du nouveau port à conteneurs entré en service en

10. La décision de mise en étude date de 1990 (Jacono, 1996) mais celle-ci ne verra le jour qu’en 1995. Entre 1990 et 1995 , les objectifs de l'étude ont considérablement évolué. À sa publication, la ZPPAUP est beaucoup plus qu'un document traitant uniquement de la restauration des façades.

11. Documentation and Conservation of Buildings, Sites and Neighborhoods of the Modern Mouvement.

12. Figaro Magazine, 22 novembre 2005, p. VII.

13. Le choix entre les deux villes (et entre les deux maires, tous les deux proches du Président de la République) n’a pas été facile. La diplomatie française a dû toutefois poser ses priorités de manière à faire passer un message clair auprès de l'Unesco. Quelques semaines avant l'annonce officielle de l'Unesco, le maire de Bordeaux a officiellement retiré la candidature de sa ville. 
2006 (Port 2000), aujourd'hui très menacé, est conçu comme un ouvrage « fort » à la « hauteur de l'œuvre architecturale entreprise par Auguste Perret ».

Dans une stratégie de «Starchitecture », le maire du Havre a fait appel à l'architecte Jean Nouvel pour la construction du futur équipement. Dans son projet pour Le Havre celui-ci ne manque pas de rendre hommage à Auguste Perret : "Les bassins, la ville construite par Auguste Perret classée au patrimoine mondial de l'Humanité, les navires, le Pont de Normandie, l'estuaire, la mer : Le Havre représente une source d'inspiration complexe et unique. Mon projet de Centre de la mer et du développement durable prend de la hauteur pour une découverte optimale de l'imbrication originale de ces atouts. Je n'aurais jamais pu réaliser Odyssey 21 et le Complexe aquatique et sportif ailleurs qu'au Havre car ils sont intimement liés à la ville ${ }^{14}$. »

Les Docks Vauban et le développement des quartiers de l'Eure et de Saint-Nicolas ont déplacé les initiatives vers l'Est de la ville en étoffant son offre ludique et touristique : conçus par les architectes Reichen \& Robert, maîtres de la réhabilitation industrielle, dédiés au commerce et aux loisirs dans d'anciens docks portuaires, ils sont complétés par le centre aquatique, ludique et sportif imaginé par Jean Nouvel. Les acteurs locaux puisent leurs références dans les waterfronts requalifiés des villes américaines telles que Baltimore... Ces ambitions urbaines ont incontestablement été encouragées par l'optimisme qui a soufflé sur la ville au milieu des années 2000 alors que Le Havre se trouvait sous les projecteurs de l'Unesco.

\section{La valorisation du patrimoine Perret}

Depuis l'inscription au patrimoine mondial, plusieurs actions ont été mises en place par la municipalité visant à mettre en valeur et à mettre en visite, voire en tourisme, le patrimoine Perret. Ces initiatives s'adressent aussi bien à un public local et régional que touristique. Elles reposent sur une véritable pédagogie du regard et du sentiment, destinée à expliquer l'intérêt, la spécificité, voire l'exemplarité de ce patrimoine méconnu. Les enjeux sont de taille : le patrimoine Perret constitue le centre même de la ville et est, en ce sens, lié à de véritables enjeux d'affirmation d'une centralité urbaine de référence pour l'ensemble de l'agglomération. Mais il est aussi le véhicule principal, surtout après l'inscription au patrimoine mondial de l'Unesco, de la construction d'une nouvelle image de marque.

Il convient ainsi de comprendre que les efforts de valorisation touristique du Havre, tout en ciblant un public extérieur, y compris touristique, visent également à enraciner la conscience patrimoniale localement.

Les problématiques de valorisation du patrimoine et de mise en tourisme sont effet nouvelles ${ }^{15}$ et Le Havre affiche un caractère pusillanime germé dans des années de complexe « de laideur». Traumatisés par une histoire douloureuse et n’assumant pas le patrimoine Perret, les Havrais ont cultivé un sentiment de « honte » nourri par des « regards extérieurs » méprisants. Aussi, prendre confiance dans le potentiel attractif de la ville, assimiler le centre reconstruit comme patrimoine d'exception et en assumer la singularité sont apparus comme des enjeux auprès de la population locale en premier lieu.

Car bien que Le Havre moderne témoigne des aspirations sociales et urbaines de l'aprèsguerre et qu'il soit également remarquable par ses qualités architectoniques et innovantes ${ }^{16}$,

14. [http://www.havre-developpement.com/upload/Un_avenir_d_exception.pdf].

15. Le premier élan de l'activité touristique du Havre s'est construit sur un patrimoine naturel, la mer, comme destination balnéaire du XIX ${ }^{\mathrm{e}}$ siècle. À l'instar de ses voisines de la côte normande : Honfleur, Deauville, Cherbourg, Dieppe ou Étretat, Le Havre a connu une période faste où se sont édifiés des établissements de bains, des hôtels de luxe, et des casinos. Mais peu avant la première guerre mondiale, l'activité balnéaire décline, minée par la concurrence des plages de BasseNormandie et par les travaux d'agrandissement du port. Le Havre industriel, renommée « Bouville » (bout du monde) par Sartre (dans La Nausée, 1938), ne présente alors plus d'intérêt pour la visite. Par la suite, la destruction du centre et la reconstruction moderne ne feront que conforter l'image d'une ville inhospitalière.

16. Systématisation de la trame à module, développement de la préfabrication, exploitation des potentialités du béton, etc. 
l'architecture moderne en France reste, toujours aujourd'hui, victime d'un déficit d'image. Parce qu'elle porte la connotation d'un échec de l'architecture « de tours et de barres » et qu'elle se fait symbole d'une société qui bâtit les grands ensembles, l'architecture moderne véhicule une image négative (Andrieux et Chevalier, 2002). Le béton lui-même, matériau brut et « gris $^{17}$ » évoquant « ascétisme, inhumanité » et «style-usine » (Andrieux et Chevalier, 2002) porte une valeur anti-patrimoniale.

Du fait de ces connotations et préjugés généralisés, l’inscription au patrimoine mondial n'a pas immédiatement balayé les héritages lourds et négatifs qui pesaient sur Le Havre. Par conséquent, l'effet Unesco a fonctionné comme un argument fort, à la fois interne et externe, mais une fois l'inscription obtenue, les acteurs locaux ont dû inventer les moyens et outils pour tirer pleinement profit de toutes les possibilités (pas encore connues, exploitées et maitrisées) que celle-ci est susceptible d’offrir au Havre.

\section{Conserver et protéger le patrimoine Perret, patrimoine mondial de l'Unesco}

La valorisation du patrimoine Perret s'appuie sur des dispositifs mis en place pour accompagner la pérennité de l'architecture et sa conformité aux principes de son créateur.

En effet, les principes de l'architecture de Perret sont souvent ignorés de la population, malgré la ZPPAUP et le travail de pédagogie effectué depuis son instauration. Le béton repeint, l'installation de doubles vitrages ou de stores fantaisistes, etc., témoignent des difficultés de compréhension de ce patrimoine. Si ces distensions entre services du patrimoine et habitants sont fréquentes dans d'autres villes, elles dénotent, dans le cas du Havre, des difficultés inhérentes en termes de compréhension, voire d'acception, au patrimoine Perret.

Dans ses efforts de protection et de valorisation, la ville s'appuie sur des dispositifs ad hoc ou réinventés. Le FISAC ${ }^{18}$, fond d'aide conçu pour le soutien des commerces de proximité des quartiers excentrés a été exceptionnellement utilisé après l'inscription au patrimoine mondial (dès 2006) dans le centre-ville du Havre, pour subventionner les travaux de rénovation. Les commerçants sont accompagnés financièrement et techniquement dans leurs démarches mais sont alors contraints de respecter les mesures de protection et de mise en valeur, le projet étant soumis à l'Architecte des Bâtiments de France.

Principalement dédié à l'aide directe aux commerces, le FISAC a permis notamment de réaliser en une seule phase le linéaire piéton de six restaurants rue Racine, face au Volcan de Niemeyer (figure 1).

Rue de Paris, une mesure particulière a été engagée pour l'uniformisation des arcades par des stores entre-poteaux dont les couleurs sont fixées par îlot ${ }^{19}$. Même si cela peut paraître paradoxal, le dispositif FISAC est à mettre en rapport avec l'inscription au patrimoine mondial. C'est effectivement cet argument qui a permis d'y faire appel dans le cadre du secteur protégé et c'est le Fond d'intervention pour les services, l'artisanat et le commerce dont la vocation est a priori autre, qui a facilité la valorisation du patrimoine Perret par l'intermédiaire,

17. Cette qualification du béton « gris » revient à plusieurs reprises, lorsque les visiteurs évoquent leur a priori sur Le Havre. La réalité des multiples couleurs apportées par les traitements du béton (bouchardé, lavé ou brut) est alors " montrée » par le guide. Observation, «ballade Perret », le 13 mars 2010.

18. Le Fond d'intervention pour les services, l'artisanat et le commerce (FISAC) du « centre reconstruit, patrimoine Unesco » a été accordé en 2006 pour une durée d'environ trois ans avec une enveloppe de 4,2 million d'euros. Les commerces du périmètre défini peuvent être subventionnés à hauteur de $40 \%$ (avec un plafond à 20000 euros) pour la réalisation des travaux extérieurs (vitrine, terrasses, grille). Cependant, le FISAC ne permet pas de subventionner les travaux d'aménagement intérieur. 101 commerces du périmètre Unesco ont bénéficié de subventions (figure 1). Deux autres FISAC sont en cours dans les quartiers Nord et Sud, dans un cadre correspondant plus à la vocation originelle de ce fond d'aide aux commerces en difficulté. Leurs budgets s'élèvent respectivement à environ 1,9 million d'euros.

19. Leur subventionnement à $50 \%$ n’a pourtant pas décidé les commerçants à les mettre en place car ils se révèlent être une source de conflit entre les commerces et les logements situés aux entresols. Cependant, cette mesure va prochainement faire l'objet d'une nouvelle campagne de sensibilisation. 
Maria Gravari-Barbas, Cécile Renard

par exemple, de la mise en évidence des éléments structurels (poteaux, poutres) des linéaires commerciaux.

De manière plus générale, le Service de l'urbanisme de la ville, en charge de la ZPPAUP, voit son travail «facilité » depuis l'inscription Unesco en 2005 car le respect du règlement est «mieux accepté ${ }^{20} »$. Bien que n'ayant pas de portée juridique, l'inscription au patrimoine mondial fonctionne ainsi comme un argument qui permet de communiquer localement; invoquer le mondial permet en ce sens de mieux expliquer les enjeux du local : opter pour une mauvaise couleur de store ou peindre une colonne en béton apparent ou encore changer une menuiserie, apparait effectivement comme une atteinte aux valeurs universelles patrimoniales et offre un argumentaire légitimant aux techniciens de la ville.

La valorisation du périmètre du patrimoine mondial repose également sur un nouveau plan lumière. Celui-ci a une portée plus générale, dont l'accompagnement du trajet du tramway, mais se concentre en particulier dans le périmètre du centre-ville reconstruit [ambiance lumineuse de la rue de Paris traitée comme un ensemble cohérent; de la place de l'Hôtel de Ville au quai Southampton; de la passerelle du Commerce et son environnement (bassin du Commerce, bord à quai, Casino, etc.)]. Le patrimoine Perret se donne ainsi à voir de jour et de nuit.

\section{Perret mis EN Visite : Une SENSIBILISATION}

La progressive patrimonialisation a vu se développer des produits touristiques orientés vers la découverte du centre reconstruit. La prise de conscience de la nécessité de structurer la valorisation et l'accès au patrimoine Perret mais également son potentiel attractif a été progressive depuis l'instauration de la ZPPAUP.

Cependant, la mise en visite effective a été largement développée avec le label «Ville d'Art et d'Histoire » dès 2001. Dans ce cadre, le travail de l'animatrice du patrimoine s'est concentré sur la problématique de l’identité Havraise et a pour objectif de faire «oublier le mot devenu péjoratif et flou de "reconstruction" au profit des termes "modernes" et "années 50”». (Chauvin, 2002).

C'est dans cette démarche que s'est inscrite l'une des premières mesures de «ville d'A + H ». Cherchant à valoriser le patrimoine Perret mis en relation avec le quotidien des années 1950, la ville a souhaité acquérir un appartement dans l'un des ISAI ${ }^{21}$ conçus par Perret lui-même sur la place de l'hôtel de ville. En 2005, dès que l'un de ces appartements s'est libéré, la ville l'a préempté et a ainsi pu mettre en place un outil pédagogique original : l'appartement témoin : «L'appartement témoin Perret, par sa dénomination et les partis scientifiques qui le sous-tendent, s'assume comme une interprétation. Ce "micro-musée" a l'ambition de présenter une synthèse des règles de l'habitation au temps de la Reconstruction; un modèle-type, conçu principalement à partir d'éléments mobiliers locaux et authentiques, qui a pour mérite principal d'éclairer la spécificité d'un modèle pédagogique d'“habiter", proposé sur la scène Havraise. Cette interprétation est dépersonnalisée, puisqu'elle ne repose pas sur la "microhistoire" d'une famille particulière mais sur un idéal du logement "ordinaire”, tel qu'il était véhiculé à l'époque dans les revues ou les expositions traitant de l'espace de l'habitation » (Chauvin, 2007).

Véritable reconstitution d'un intérieur de l'après-guerre (objets et mobilier d'époque chinés dans la région ou dons de Havrais), l'appartement témoin permet d'aborder à la fois l'évolution sociétale et ménagère mais aussi d'expliciter le plan des logements, l'ingéniosité et l'innovation dans la modularité des espaces. Depuis sa mise en visite, l’appartement se révèle particulière-

20. Entretiens menés auprès du service d'urbanisme en mars 2010.

21. «Immeubles sans affectation individuelle », destinés à la location. Ce sont les premiers immeubles « sortis de terre ", achevés en 1947. Bien que le plan d'ensemble ait été élaboré par l'atelier Perret, seul ces quelques îlots ont été dessinés par l'architecte. Les autres ont été confiés à des architectes plus jeunes, souvent élèves de Perret. Aussi l'enjeu de l'appartement témoin était-il de montrer un véritable logement «signé » par Perret. 
ment attractif, séduisant des visiteurs «spécialisés » (amateurs d'art, de design, d'architecture) mais aussi un public plus large, sensibilisé au patrimoine Perret grâce à cet espace facilement appropriable. Pour le public parfois non acquis à la cause Perret, l'appartement permet en effet d'humaniser ce patrimoine ${ }^{22}$. Ainsi, l'appartement témoin est aussi le support privilégié de manifestations spécifiques, tels que «Pièces de vies », mêlant des interventions artistiques avec du discours social. Ce spectacle mené par une comédienne consiste en une investigation de la mémoire Havraise de la reconstruction, au travers d'anecdotes issues de témoignages et est ponctué par une approche historique d'un guide-conférencier.

En 2009, plus de 10000 personnes ont visité l'appartement Perret (contre 3600 environ en 2006), dont la moitié non originaire de la région ${ }^{23}$.

Si ces activités rencontrent un grand succès, cela démontre la demande des visiteurs de comprendre ce patrimoine difficile d'abord. Dans cette optique, les services de la «Ville d'Art et d'Histoire » ont acquis en 2010 un local rue de Paris, en plein cœur du centre reconstruit (fig. 1), pour y localiser un «centre d'interprétation du patrimoine ». Lieu dédié à Perret (information, expositions), vitrine des activités et ballades proposées, cette structure permet d'améliorer la logistique en tant que lieu identifié et point de départ des visites.

La question de la visibilité et de l'abord direct du patrimoine par les visiteurs a également motivé la mise en place de 27 cartels, panneaux explicatifs directement liés au circuit Unesco, dont l'inauguration a eu lieu en 2010. Cette signalétique extérieure est destinée à rendre la visite plus autonome et l'identification du logo Unesco sur les supports devrait renforcer la visibilité de la labellisation.

Parallèlement à ces lieux pérennes, plusieurs visites ont été mises en place pour donner à voir le patrimoine Perret au public, qu'il s'agisse de résidents ou des visiteurs extérieurs. La «ballade Perret », au départ du musée Malraux pour s'achever à l'appartement témoin, est celle qui inscrit le mieux le patrimoine Perret dans son contexte. Le départ de la visite offre des vues sur le port qui permettent au guide-conférencier d'évoquer Le Havre industriel et l'importance de l'activité portuaire. Les vues sur le quartier Saint François permettent d'introduire la question du « choix » de la reconstruction, à l'identique ou non, et au loin le développement des Docks Vauban permet une ouverture sur le renouvellement contemporain du Havre. La visite se poursuit par la découverte du patrimoine Perret suivant le circuit du «triangle Perret » par le boulevard François $1^{\mathrm{er}}$ puis l'avenue Foch pour arriver place de l'Hôtel de Ville et terminer à l'appartement Perret (fig. 1).

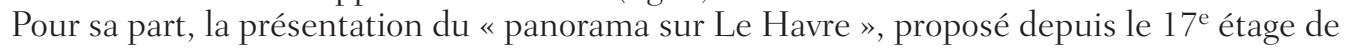
l'hôtel de ville, offre une sensibilisation à l'urbanisme et à l'histoire du Havre.

Si le dispositif de visites existant aujourd'hui au Havre n'est pas exempt de reflexes de mise en tourisme plus génériques, tel que le «petit train touristique » identique à celui qui sillonne les rues de nombreuses autres villes, il dénote certainement d'une volonté de réflexion approfondie sur des dispositifs médiatiques conçus de façon ad hoc pour ce patrimoine issu de la modernité et de la reconstruction.

\section{Célébrer Perret}

La communication sur l'inscription utilise, en interne et en externe, tous les canaux possibles. Les panneaux signalétiques posés aux entrées de la ville signalent aux visiteurs qu'ils pénètrent dans le périmètre Unesco et revendiquent l'appartenance du Havre au club prestigieux des villes du patrimoine mondial.

22. L'une des critiques récurrentes du patrimoine Perret concerne la froideur des bâtiments « de l'extérieur ». Lors de la visite de l'appartement témoin, les visiteurs opposent souvent le confort intérieur « inattendu » au regard de ce qu'évoquent les bâtiments extérieurement. Données issues de l'observation faite dans le cadre des visites de l'appartement témoin le 14 mars 2010.

23. Données Ville d'Art et d'Histoire. 
Maria Gravari-Barbas, Cécile Renard
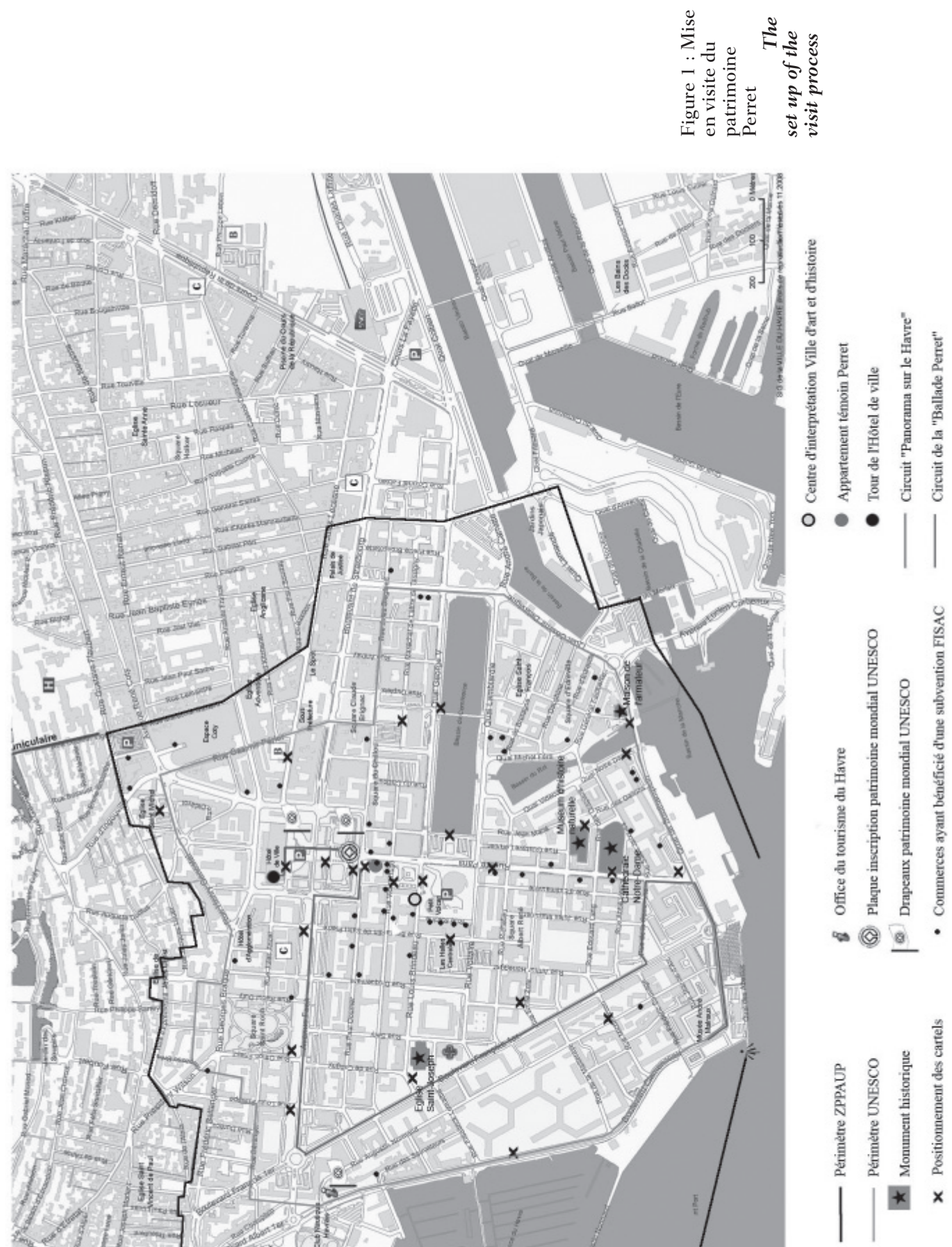
Sur le site internet de l'office de tourisme du Havre, quatre bandeaux défilent : «Le Havre, berceau de l'impressionnisme », "Le Havre, une ville verte et durable », «Le Havre sur mer » et «Le Havre, patrimoine mondial de l'humanité ». La référence au patrimoine mondial est systématiquement présente dans les brochures sur Le Havre et l'en-tête du site de l'office du tourisme annonce «Le Havre, patrimoine mondial »; à l'onglet dédié, un petit film « promotionnel » offre des vues du centre reconstruit, sans commentaire mais en musique, à côté d'un court texte qui présente le patrimoine inscrit ainsi que, très succinctement, la biographie d'Auguste Perret.

La ville utilise le label dans la communication touristique, le plus souvent en indiquant sur des photographies du centre-ville la mention « inscrit au patrimoine mondial ». Quelle que soit donc l'efficacité du label, Le Havre joue cette carte de manière volontariste.

La fierté de l'obtention du label a notamment été exprimée par l'organisation d'événements autour des anniversaires de l'inscription. En 2007, pour les deux ans, seule une animation commerciale a été proposée : distribution de flyers et de sets de table avec un visuel Unesco ${ }^{24}$. En 2008, l'événement a pris plus d'envergure. L'hôtel de ville et l'espace Oscar Niemeyer (le Volcan), bâtiments emblématiques du périmètre, ont été les supports de projections lumineuses artistiques. La mise en lumière, accompagnée de musique, s'est déroulée de mi-juin à fin septembre tous les soirs avec différents graphismes proposés. La manifestation a été relayée par la création de cartes postales représentant les différentes illuminations pour être distribuées aux visiteurs et habitants dans les commerces ${ }^{25}$. Mais c'est pour l'anniversaire de cinq ans de l'inscription, en juillet 2010, que la ville a mis en place le dispositif festif le plus important. Outre les événements organisés par la municipalité (visites, concerts, ouverture de lieux habituellement fermés, tels que certaines salles de l'hôtel de ville), sont créés des objets de collection célébrant les cinq ans de l'inscription.

\section{Une valorisation sans appropriation?}

L'analyse des projets mis en place au Havre, dans le cadre du périmètre du patrimoine mondial témoigne d'un investissement sans doute plus important des décideurs locaux que celui observé dans des villes qui ne partaient pas avec autant « d’handicaps » que Le Havre : appartement témoin, centre d'interprétation du patrimoine Perret, visites spécifiques, adaptation des moyens juridiques et urbains existants à la nature du patrimoine de la reconstruction... Les acteurs locaux ont fait preuve d'une volonté certaine de valoriser le patrimoine Perret. Leurs approches s'inscrivent dans la continuité de celles entreprises depuis les années 1990 : montrer, avec si nécessaire des « labels à l'appui », l'intérêt de l'architecture Perret, aux populations locales et extérieures - dans une approche où le changement de regard des populations locales contribue au changement de celui des populations résidentes et, inversement, l'évolution du regard des populations résidentes, ambassadeurs de la patrimonialisation du Havre, est indispensable pour attirer les visiteurs. Toutefois, ces stratégies émanent toujours « d'en haut », et sans réaction positive « d'en bas », risquent à terme de s'essouffler. Dans cette situation paradoxale, la valorisation touristique du patrimoine Perret tend à fonctionner comme le véhicule de la reconnaissance du patrimoine par la population locale.

\section{UN DISPOSITIF DE VALORISATION DU PATRIMOINE QUI ATTEINT PROGRESSIVEMENT LE PUBLIC TOURISTIQUE}

L'inscription du centre reconstruit sur la liste du patrimoine mondial a mis Le Havre sous la lumière des projecteurs. Entre les réactions critiques, hostiles à la valorisation de cet urba-

24. Cette animation a été mise en place dans le cadre du FISAC, évoqué plus haut.

25. Les illuminations de 2008 sont à initiative et financées par la ville du Havre. Quant à l'émission des cartes postales et la distribution aux commerces, elles ont été réalisées dans le cadre du FISAC. 
Maria Gravari-Barbas, Cécile Renard

nisme moderne et les plus enthousiastes qui ont porté dès le départ le projet d’inscription du patrimoine Perret, c'est bien souvent la « surprise » qui prédomine. En effet, si une partie de la population havraise a suivi la mise en patrimoine progressive du Havre « au quotidien ", ce n'est pas le cas des visiteurs pour qui l'annonce de l'inscription par voie de presse a suscité de la curiosité. «Comprendre pourquoi le centre reconstruit du Havre est inscrit sur la liste du patrimoine mondial » apparaît ainsi comme l'un des motifs de visite principalement invoqué ${ }^{26}$.

Pourtant, le label Unesco ne semble pas réellement assimilé par les visiteurs. Si la plupart peut en donner une définition approximative ("protection », «valorisation du patrimoine »), très peu d'entre eux peuvent citer d'autres sites classés ou en donner le nombre ${ }^{27}$. Beaucoup de visiteurs pensent que la labellisation est une manne financière pour Le Havre qui bénéficierait alors de subventions de la part de l'Unesco, alors qu'en réalité une inscription sur la liste du patrimoine mondial ne donne droit à aucune aide particulière.

Toutefois, alors que l'inscription suscite la curiosité qui déclenche la venue des visiteurs, aucun d'entre eux ne dit être influencé par une labellisation « patrimoine mondial » dans le choix de ses destinations touristiques en général. Si l'impact de l'inscription du Havre est effectif en termes d'attractivité touristique ${ }^{28}$, c'est principalement parce qu'elle implique une médiatisation qui renouvelle l'image de la ville.

Les plus curieux et désireux de comprendre l'inscription du patrimoine Perret sont sans nul doute les visiteurs qui ont connu Le Havre "avant ", c'est-à-dire qui sont venus une première fois au Havre il y a vingt à trente ans, alors que la ville était en rejet du centre-ville moderne. Une touriste allemande raconte que lors de son accueil dans une famille Havraise, il y a vingt ans, ses hôtes lui ont montré la plage mais ont évité le centre reconstruit jugé peu valorisant $^{29}$. Pour elle, comme pour plusieurs autres visiteurs, l'annonce de l'inscription est apparue en contradiction avec leur représentation de la ville et a suscité un intérêt et une sensibilité nouveaux pour le patrimoine Perret. Parmi ces visiteurs, tous ont constaté des efforts de communication et d'embellissement de Havre. Nombreux sont ceux qui ont trouvé la ville « plus belle » que lors de leur première visite, et dit comprendre et apprécier le centre reconstruit parce qu'il leur était expliqué. Alors qu'ils affirment s'être posé la question avant de visiter Le Havre, tous ont estimée l’inscription Unesco justifiée.

Si l'annonce de la patrimonialisation agit comme générateur de visites, les dispositifs de valorisation de l'urbanisme Perret sont indispensables pour accompagner les visiteurs en demande de « clés » pour comprendre un patrimoine difficile d'abord. La mise en place de ces dispositifs permet d'insérer Le Havre dans une offre touristique.

L'évolution des « regards extérieurs » (Urry, 2002) a été accompagnée et, à son tour, a suscité dans une relation réflexive, les discours des médias. Les guides touristiques en particulier ont peu à peu intégré comme destination le patrimoine Perret. Ainsi, en 2004, alors que la ville bénéficiait déjà depuis plusieurs années du label «Ville d'Art », le Guide du Routard introduit le chapitre sur la ville par ces mots : "Pas facile d'aborder une telle ville dans un guide touristique! Le $2^{\mathrm{e}}$ port de France, entièrement reconstruit, présente une architecture à l'évidence peu... amène. Le Havre fait partie de ces villes qu'on ne peut aborder au présent. Pour en parler et la comprendre, il faut revenir en arrière » (Josse et al., 1994). En 2009, cette même introduction prend la tournure suivante : «Naguère, on hésitait à décrire longuement une telle ville dans un guide touristique! Le $2^{\mathrm{e}}$ port français (et le $5^{\mathrm{e}}$ port européen) s'est

26. «Q - Pourquoi vous êtes venus au Havre? R - Pour comprendre pourquoi cette ville a été classée au Patrimoine Mondial de l'Unesco. Je savais que c'était classé et me suis dit que ça devait avoir un intérêt... » Entretiens avec des participants à la ballade Perret et des visiteurs l'appartement témoin, mars 2010.

27. La plupart des visiteurs à la question « combien y a-t-il de sites sur la liste du Patrimoine Mondial? » répond : « une centaine de sites ». A la question « connaissez-vous d'autres sites inscrits? », beaucoup de visiteurs ne savent pas répondre, peu d'entre eux répondent correctement en citant un ou deux sites, le plus souvent parce qu'ils les ont déjà visités.

28. En effet, en 2004 le nombre de visiteurs ayant suivi de visites guidées était de 5297 . En 2005, il a été de 9091 , en 2006 de 17000 , en 2007 de 22000 , en 2008 de 24000 et en 2009 de 27000 . Données ville A + H, 2010.

29. Entretiens avec des participants à la ballade Perret et des visiteurs l'appartement témoin, les 13 et 14 mars 2010. 
en effet contenté jusqu'au tournant du $\mathrm{XXI}^{\mathrm{e}}$ siècle de présenter une architecture à l'évidence peu... amène. "

L'évolution des discours dans le sens de la valorisation du patrimoine Perret a été progressive jusqu'en 2005, date de l'inscription sur la liste du patrimoine mondial. Au cours des années 1990 et 2000, les guides utilisent les mêmes descriptions peu valorisantes ${ }^{30}$ et l'on ne remarque pas d'évolution du discours. À partir de 2001, si l'obtention du label « ville d'Art et d'Histoire » est bien notifiée, elle ne représente pas un argument suffisant pour changer la représentation du Havre donnée par les guides. Cependant, après 2005 et la labellisation Unesco, on remarque un changement radical dans le ton et l'enthousiasme déployés à propos de la ville. Ainsi, alors qu'en 1994, comme en 1999, le Guide du routard, suggère simplement de regarder la ville « avec réalisme, un brin de condescendance et, à défaut d'une âme, [de lui découvrir] un cœur », en 2004 il déclare que la ville «mérite un effort mais aussi un détour! » et finit en 2009 par conseiller « un détour prolongé » (Josse P., Bouffet F., Lucchini B. et al., 2004, 2009).

La plupart des guides font par ailleurs explicitement référence au patrimoine mondial et arborent le logo du patrimoine mondial.

Le label patrimoine mondial semble ainsi fonctionner vis-à-vis des cibles sensibles précisément à la labellisation : si le nombre d'étoiles accordées et les commentaires faits par les guides correspondent à une sorte de «label touristique » accordé aux sites, on ne peut pas ne pas observer une certaine adéquation entre le label touristique - celui du guide - et le label patrimonial - celui de l'Unesco.

Relayée par les guides qui encouragent la visite de la ville et l'inscrivent clairement dans l'industrie du tourisme, la mutation du Havre comme destination touristique semble bien engagée et comprise de la part des visiteurs qui perçoivent l'intérêt patrimonial du centre reconstruit.

Mais de quelle manière ces évolutions des discours, producteurs de valeur ajoutée en termes d'image, induisent-elles des changements au niveau de la population locale? De quelle manière les messages vertueux envoyés aux populations du monde entier, reviennent-ils, par un effet de boucle, redorer localement l'image du Havre?

\section{Un « médiateur » De L’appropriation du patrimoine Perret par les Habitants locaux?}

L'appropriation du patrimoine Perret par la population locale apparaît en effet plus sensible et ambigüe. L'étude sociologique réalisée par le CERIS (Monjaret et al., 2010) montre une « ambivalence » du sentiment des Havrais à l'égard de leur ville : ils sont à la fois fiers de leur ville-patrimoine mondial mais encore mal à l'aise avec la «froideur » et la trame linéaire de son urbanisme moderne. L'étude montre d'ailleurs la manière dont plusieurs anciens habitants du Havre, ayant vécu la reconstruction, ont des difficultés à la comparer avec d'autres sites urbains du patrimoine mondial : «C'est ridicule... mais il ne faut pas le dire à monsieur le Maire... Mais quand vous voyez des villes comme Athènes, ou, ça n'a rien à voir... Moi si j'avais de l'argent, je mettrais tout par terre et je mettrais autre chose. [...] Je trouve ça lamentable, on a vécu là-dedans, c'était pas mal, confortable mais quand on voit qu'on compare ça à Angkor, c'est lamentable, je ne sais pas comment il a attrapé ça, mais c'est n’importe quoi ! [...] Quand on voit Vienne, Prague, Budapest, trois villes, qui sont autre chose que Le Havre » (Monjaret et al., 2010).

La labellisation Unesco semble pourtant avoir fait évoluer les perceptions et il ressort de l'enquête du CERLIS que même si les sentiments « on aime » ou « on n'aime pas » sont bien

30. Exemple du Guide du pays de Caux de 1988 et de 1999. Dans ces deux versions, le texte de présentation du Havre n’a pas évolué. Le passage sur le centre reconstruit est très court et peu encourageant. Bien que les descriptions essayent de donner une bonne image de la ville, on ressent une réticence à conseiller réellement la visite du centre reconstruit, la vision de l'œuvre de Perret est révélée à travers cette question posée « Mais l'équipe Perret ne chercha-t-elle pas davantage à faire prévaloir un point de vue d'esthète ou d'architecte plutôt que d'un point de vue d'urbaniste ou de sociologue? » (Auger et Garnier, 1988, 1999). 
présents, la critique tend aujourd'hui à s'estomper avec l'attribution du label. Certains Havrais sont bien conscients des efforts d'esthétisation réalisés par la ville et déclarent désormais l'apprécier et la trouver «belle». «Autant Le Havre, quand j'étais petite, je trouvais que c'était pas très beau, c'était gris, mais depuis quelques années, ça a beaucoup évolué. La ville s'est embellie [...] c'est toujours les mêmes constructions, je suis d'accord, mais il y a eu des efforts, par exemple pour la gare, l'université, le bord de mer... Beaucoup de choses ont été améliorées ${ }^{31}$. "

Pour eux, Le Havre d'aujourd'hui offre un nouveau visage et leur demande un effort d'appréhension d'un patrimoine qui ne leur avait jamais été expliqué. Une habitante du Havre, participant à la ballade Perret explique : « Même les Anglais ne s'arrêtaient pas au Havre. On passait c'est tout. Beaucoup de gens disaient Le Havre, c'est moche. Alors que maintenant, enfin quand on connaît... Parce que nous, petits, on ne nous expliquait pas vraiment tout ce qui a été fait. Donc c'est pour ça que je viens visiter. Parce que même en y passant tout le temps, y’a des choses que je ne connaissais pas alors que j’y suis née ${ }^{32}$. »

Ainsi, bien que certains Havrais s'intéressent au patrimoine Perret et font l'effort de participer aux animations proposées, leur appropriation apparaît beaucoup plus compliquée que celle des visiteurs.

La difficile valorisation symbolique est à mettre en parallèle avec une très timide valorisation économique du patrimoine. Ainsi, même si l'appartement Perret mis en visite fonctionne comme un outil stratégique de l'explication de l'architecture Perret et de la médiation de sa valeur patrimoniale, les « vrais » appartements Perret, disponibles dans le marché, n'ont pas connu une valorisation notable. D’après des entretiens effectués auprès de différentes agences immobilières (mars-avril 2010), la signature Perret ne constitue pas réellement, pour les Havrais, une plus-value culturelle pour laquelle ils seraient prêts à acquitter une plusvalue financière. En effet, si certains Havrais demandent des logements dans des immeubles construits par Perret, c'est avant tout parce qu'ils apprécient leur localisation centrale et en second lieu parce qu'ils ont pris conscience (peut-être grâce à l'appartement témoin ?) de la flexibilité dans l'aménagement intérieur qu'offrent ces appartements. Les non-Havrais seraient davantage motivés par la volonté d’investir dans un appartement "signé ». Si les agences affichent la mention «Perret » dans la présentation des biens, c'est dans l'intention d'informer (puisque cela indique un secteur et une période de construction mais aussi un mode de chauffage) mais nullement pour les valoriser.

Ce sont donc bien ces « regards extérieurs » qui font évoluer les perceptions locales de la ville. Ainsi, à travers les reportages dans la presse ou à la télévision à l'occasion de l'inscription Unesco, les Havrais découvrent leur ville « en miroir ${ }^{33}$ ». C'est à travers les médias que le patrimoine Perret leur a parfois été « expliqué » pour la première fois.

\section{L'INSCRIPTION AU PATRIMOINE MONDIAL DE L'UNESCO, UN LABEL TROP LOURD À PORTER LOCALEMENT?}

L'approche «top down » a prévalu également en termes de reconnaissance associative. La césure entre l'appréciation locale et l'appréciation nationale, voire internationale, du patrimoine est en effet lisible également en termes associatifs.

31. Entretiens avec des participants à la ballade Perret et des visiteurs l'appartement témoin, mars 2010.

32. Idem.

33. «J'ai vu sporadiquement la reconstruction, on ne s'y intéressait pas, on la jugeait affreuse à l'époque, c'était du béton, béton. J'ai acheté un bouquin à une de mes petites filles qui fait des études d'architecture sur Perret. Perret il a jubilé, il avait l'espace libre. Il a conçu, il y a une chose intéressante, autant on finit par s'y habituer à la reconstruction, autant Le Havre n'est pas très joli du sol, autant en vue aérienne, c'est superbe. J'ai vu des photos, il y a des arrivées du Tour de France au Havre, ils venaient de Caen et passaient par le Pont de Normandie. Un autre Tour, j'habitais rue de Paris, avant la rue Guénot qui était à côté du Square Saint-Roch, il passait sous mes fenêtres. Lémission Thalassa passée il n’y a pas longtemps. C'est de voir tout le projet de Perret, on voit que ça répond à un plan. » (Monjaret et al., 2010). 
Ainsi, l'investissement associatif des Havrais témoigne toujours aujourd'hui de la difficulté à prendre en compte le patrimoine de la reconstruction. Aucune association de protection du patrimoine Perret ou, de manière plus générale, de l'architecture moderne et de la reconstruction n'a émergé au Havre au cours des dernières années. Ceci est à mettre en rapport avec les nombreuses associations qui œuvrent pour la connaissance et la sauvegarde du patrimoine maritime et portuaire. Ainsi, l'association Amis des paquebots et marine marchande et l'Association du Musée maritime et portuaire du Havre, ont toutes deux pour mission la conservation et valorisation du patrimoine maritime et portuaire Havrais. Certaines associations sont encore plus spécialisées, comme l'Association French Lines, qui a pour objet de conserver et valoriser le patrimoine des compagnies maritimes françaises. Quant à l'Association Remorqueur US ST : 488-Le Havre 16th port et l'Association de l'Hirondelle de la Manche, elles sont toutes deux centrées sur la restauration et la remise en eau de deux navires classés Monument Historique. Enfin, l'Adias ACH est l'association de sauvegarde des collections des anciens Ateliers et Chantiers du Havre. Outre la préservation du patrimoine maritime, une seule association est dévolue au patrimoine architectural Havrais (Mémoire et patrimoine) mais se concentre sur la période 1939-1945.

Si l'attachement associatif est un critère de l'appropriation patrimoniale, le patrimoine Perret ne semble pas faire partie des préoccupations ni de l'affectif des Havrais.

A contrario, ce sont les associations internationales qui, les premières, se sont penchées sur le cas du Havre et qui ont été des partenaires incontestables de sa reconnaissance officielle. Le noyau de cette reconnaissance fut l'Association DoCoMoMo ${ }^{34}$ dont l'action vise précisément à reconnaître l'architecture du mouvement moderne. L'association a probablement joué un rôle important dans la médiation de l'architecture Perret auprès de la population locale. Mais comme l'affirmait, à juste titre en 2005 Fabienne Chevallier, son ancienne présidente : «Une association comme DoCoMoMo ne peut pas se situer seulement dans le champ de l'appropriation du patrimoine par la population locale. [...] Leur architecture quotidienne [des habitants locaux] se rattachait à une histoire nationale de longue durée. Ils m'ont beaucoup remerciée pour cette prise de conscience. Nous ne sommes pas des historiens locaux; nous ne pouvons pas agir sur le travail de mémoire au quotidien, mais seulement y contribuer. »

Aujourd'hui, ce sont les instances de la ville qui veillent sur la sauvegarde et la reconnaissance du patrimoine Perret. Celui-ci, protégé - symboliquement et juridiquement - par une superposition de procédures et de périmètres (périmètres M.H., ZPPAUP, périmètre Unesco), est peu menacé, probablement bien moins que d'autres biens construits à une période antérieure $^{35}$. Ainsi, le patrimoine Perret n'a pas été caractérisé par des conflits patrimoniaux susceptibles de générer la création de nouvelles associations. Une éventuelle menace pesant sur les immeubles du centre reconstruit pourrait avoir un effet déclencheur du réveil associatif qui aujourd'hui ne semble pas avoir des raisons pour militer.

Est-ce la prise en main du patrimoine Perret par la municipalité et les instances nationales qui a freiné l'éveil associatif? C'est possible. L'explication fournie par Elisabeth Chauvin, animatrice du patrimoine, nous semble toutefois plus plausible : elle met l'accent sur ce passé encore proche, non distancié, trop palpable pour qu’il soit patrimonialisé. Les efforts de ville d'Art et d'Histoire se concentrent ainsi sur la volonté de créer un imaginaire plus « rétro », très visible dans l'aménagement de l'appartement témoin et les ouvrages publiés (Ville du Havre, 2007), cherchant à positionner le patrimoine Perret là où il appartient, c'est-à-dire au siècle passé.

34. Le Havre adhère au DoCoMoMo en 1992.

35. Ainsi, l'imposant bâtiment de la Poste centrale du Havre construit vers 1920 par l'architecte du gouvernement Louis Chifflot, assisté de Pierre Chirol, « réhabilité » en logements haut de gamme a subi une opération de « façadisme », c'est-à-dire un évidage intérieur complet pour ne conserver que les façades ouvragées malgré son inscription à l'inventaire général des monuments historiques depuis 1996. 


\section{Conclusion}

Le cas du Havre est emblématique des limites d'une construction patrimoniale instrumentalisée par les autorités locales, à la fois sur le plan de ses réussites - nombreuses - et de ses limites.

Les entretiens avec les acteurs locaux, les habitants locaux et les visiteurs du Havre (régionaux ou nationaux) montrent clairement qu'il y a un " avant » et un "après " l'inscription au patrimoine mondial. L'inscription a fonctionné à la fois comme un facilitateur de lancement des nombreux projets qui ont vu le jour depuis 2005, comme un régulateur des relations municipalité-habitants - en particulier pour faire accepter un certain nombre de réglementations - et comme un facteur de création d'image, à la fois à consommation interne et externe.

Le caractère instrumental de la patrimonialisation du Havre ne pose-t-il pas toutefois les limites de cette même patrimonialisation? La désaffection des élites locales ne constitue-telle pas un frein à la patrimonialisation, dès lors que celle-ci doit aller au-delà de l'approche technique et gestionnaire adoptée depuis 1995?

L'appropriation du patrimoine Perret apparaît ainsi toujours fragile aujourd'hui. La Municipalité reste l'animateur principal des initiatives patrimoniales, l'acteur essentiel de la patrimonialisation, de son évolution et de ses dynamiques. Le Havre s'offre à nous comme un cas d'étude, particulièrement intéressant, d'un double processus instrumentalisé : la création d'un objet patrimonial international dans un premier temps; l'invention d'un modus vivendi local pour ce même patrimoine dans un deuxième temps. Dans ce processus de l'appropriation par les locaux, ce sont de nouveau des populations extérieures qui servent de "véhicule » de la valeur patrimoniale des lieux. La mise en tourisme apparaît ainsi comme une stratégie susceptible d'apporter, bien au-delà des retombées économiques encore incertaines, l'effet d'entrainement nécessaire pour l'appropriation du patrimoine Perret par la société locale.

\section{Bibliographie}

Аввам J., 1997-1998. Un patrimoine architectural et urbain exceptionnel. Les grandes opérations de la reconstruction du Havre, Faces, n 42-43, p. 10-15.

Andrieux J.-Y., Chevallier F., 2002. La réception de l'architecture du mouvement moderne, septième conférence internationale de DoCoMoMo, Paris, 16-19 septembre 2002, Institut des études régionales et des patrimoines, Palais de l'Unesco, 477 p.

Auger P., Garnier G., 1988. Le guide du pays de Caux, Paris, La Manufacture.

Auger P., Garnier G., 1999. Le guide du pays de Caux, Paris, La Renaissance du Livre.

BAnZet E., 2001. Le Havre rompt avec la nostalgie du «Quai des brumes », Le Monde, 2 février.

Barot S., 1994. Archéologie d'une reconstruction, l'exemple du Havre, dans Dieudonné P. (dir.), Villes reconstruites, du dessin au destin, actes du deuxième colloque sur les villes reconstruites, p. 108-115.

Chauvin É., 2002. Rapport d'activités, ville d'Art et d'Histoire.

Chauvin É., Gencey P., 2007. «L'appartement témoin Perret » au Havre. Un idéal moderne et démocratique au service d'une œuvre urbaine globale, Histoire Urbaine, n² 20, p. 39-54.

Colliard D., 1998. Reconstruire Le Havre, 2 : du deuil à la célébration de l'héritage, Entretien, du 16 janvier 1996, dans Andrieux J.-Y., Seitz F. (dir.), Pratiques architecturales et enjeux politiques, Paris, Picard, p. 66-79.

Galinon-Mélénec B., 2007. Voiles et dévoilement du Havre, Revue Communication et organisation, "La ville dans tous les sens ", n 32 , p. 64-79.

Gargiani R. 1996. Le Havre de Auguste Perret, ville idéale du béton armé, Faces, p. 18-22.

Gravari-Barbas M., 2002. Tourism Policies in French-post-2 ${ }^{\text {nd }-W o r l d-W a r ~ r e c o n s t r u c t e d ~ c i t i e s ~: ~ S a i n t-~}$ Nazaire, Le Havre and Lorient, Chalenges in City Tourism Research and Practice, Vienne, p. 251-264.

Gravari-Barbas M., 2003. The Challenge of Le Havre : developing tourism in a reconstructed city, Tourism, $n^{\circ} 2$, p. 141-155. 
Gravari-Barbas M., 2004a. Le cas d'Angers et du Havre. Quelle marge de manœuvre pour une « gouvernance patrimoniale »?, Pouvoirs Locaux, $\mathrm{n}^{\circ}$ 63, Les nouveaux espaces du patrimoine, p. 84-93.

Gravari-Barbas M., 2004b. Patrimonialisation et réaffirmation symbolique du centre-ville du Havre. Rapports entre le jeu des acteurs et la production de l'espace, Les Annales de Géographie, nº 640, p. 588-61 1.

Gravari-Barbas M., 2006. Image, Heritage and Identity Policies in a French-post World War II rebuilt city : the case of Le Havre, World Heritage by Unesco, communication présentée au colloque "Urban Changes in different scales : systems and structures", Université de Santiago de Compostella, Commission IGU on Monitoring Cities of Tomorrow, Santiago de Compostella, e $^{\mathrm{er}}-6$ août 2006.

Gravari-Barbas M., 2008a. Le Havre : du traumatisme de la reconstruction à l'inscription au patrimoine mondial de l'Unesco, dans Hérin R. (dir.), De la Ville perdue à la ville retrouvée, la ville en devenir, Presses universitaire de Caen, p. 265-286.

Gravari-Barbas M., 2008b. Le patrimoine au secours du développement urbain. Le patrimoine « Perret » au Havre, dans Leriche F. et al., l'Economie culturelle et ses territoires, p. 331-344.

Gravari-Barbas M., Jacquot S., 2008. Les impacts socio-économiques de l'inscription d'un site sur la liste du patrimoine mondial : une revue de la littérature, dans Prud'homme R. (dir), Les impacts socio-économiques de l'inscription d'un site sur la liste du patrimoine mondial : trois études, Unesco, centre du patrimoine mondial.

Jacono G., Arnould P., 2000. Le Havre, paysage urbain et patrimoine. Entre béton, discours et images. De la reconstruction physique à la (re)construction mentale, Paysages Urbains, Cahiers de la Méditerranée, $\mathrm{n}^{\circ}$ 60, p. 109-131.

JaCONO G., 1996. Le patrimoine d'une ville reconstruite : Le Havre, de la nécessité de la reconnaissance au mirage urbain de l'image, mémoire de DESS Université Paris 8, 189 p.

Josse P., Bouffet F., Lucchini B. et al., 1999. Le guide du routard Normandie 1999-2000, Hachette.

Josse P., Bouffet F., Lucchini B. et al., 2004. Le guide du routard Normandie 2004-2005, Hachette.

Josse P., Keravel A., Lucchini B. et al., 2009. Le guide du routard Normandie 2009-2010, Hachette.

Martin-Chauffier G., 2005. L'Unesco est-elle tombée sur la tête?, Paris Match Magazine, 11 août.

Maubant C., 1994. Patrimoine et développement local, Le Havre, 30 p.

Montjaret A., Dessajan S., Stevanovic J., 2010. Collecte des témoignages oraux, Le Havre. Les habitants du Centre reconstruit; La génération de la guerre, CERLIS, Paris.

Urry J., 2002. The tourist gaze, (1 édition 1990), Sage, Londres, 183 p.

Cet article a été reçu le 8 mai 2010 et définitivement accepté le 17 décembre 2010. 